\title{
Lidil
}

Revue de linguistique et de didactique des langues

$64 \mid 2021$

Le passif dans la langue parlée

\section{Étude sémantique des restrictions distributionnelles du passif périphrastique en français à partir d'un corpus oral}

The Periphrastic Passive in French and Distributional Gaps: A Semantic Study Based on an Oral Corpus

Pierre Jalenques

URL : https://journals.openedition.org/lidil/9849

DOI : $10.4000 /$ lidil.9849

ISSN : 1960-6052

Éditeur

UGA Éditions/Université Grenoble Alpes

Édition imprimée

ISBN : 978-2-37747-315-1

ISSN : $1146-6480$

Référence électronique

Pierre Jalenques, «Étude sémantique des restrictions distributionnelles du passif périphrastique en français à partir d'un corpus oral », Lidil [En ligne], 64 | 2021, mis en ligne le 01 novembre 2021, consulté le 24 novembre 2021. URL : http://journals.openedition.org/lidil/9849 ; DOI : https://doi.org/ 10.4000/lidil.9849

Ce document a été généré automatiquement le 24 novembre 2021.

(c) Lidil 


\section{Étude sémantique des restrictions distributionnelles du passif périphrastique en français à partir d'un corpus oral}

The Periphrastic Passive in French and Distributional Gaps: A Semantic Study

Based on an Oral Corpus

Pierre Jalenques

\section{Introduction}

Nous reprenons l'étude du passif, en français, sur le plan sémantique, à partir de données issues du français oral. Il s'agit ici de présenter nos premiers résultats, à partir $\mathrm{du}$ corpus des ESLO ${ }^{1}$. Nous nous intéressons ici à l'objet linguistique, parfois appelé passif périphrastique ${ }^{2}$, répondant aux trois caractéristiques suivantes :

- dimension syntaxique :

- le second argument du verbe (noté Y) est en position de sujet syntaxique,

- le premier argument (noté $\mathrm{X}$ ) occupe une position syntaxiquement périphérique ;

- dimension lexicale : présence de l'élément être ;

- dimension morphologique: le verbe suivant l'élément être apparait sous la forme de participe passé.

\begin{tabular}{|l|l|l|c|c|c|}
\hline \multicolumn{4}{|l|}{ (1) } \\
\hline & on peut pas dire & ils & ont été bien reçus par & tout le monde & oui oui \\
\hline & & Y & & X & \\
\hline
\end{tabular}


2 Par abréviation, nous parlerons de phrase active ou d'actif pour désigner un emploi de verbe en construction transitive directe; et nous parlerons de passif pour désigner spécifiquement une phrase au passif périphrastique.

3 Comme le soulignent Hamma et coll. (2017) et Hamma (2020), le passif en français a été étudié essentiellement à partir de données issues de l'introspection ou de corpus écrits. Or, il apparait que, sur le plan pragmatico-discursif, l'analyse de données issues de corpus oraux fournit des résultats sensiblement différents quant aux propriétés traditionnellement attribuées au passif. En particulier, selon Hamma (2020), les données orales contredisent les hypothèses courantes selon lesquelles le passif mettrait en saillance le second argument du verbe (le sujet du passif) ou bien marquerait la mise à l'arrière-plan du premier argument du verbe (le cpl. d'agent) ; selon l'auteur, le passif marquerait avant tout la mise en saillance du prédicat lui-même.

On peut dès lors se demander si, pour les propriétés proprement sémantiques du passif, l'analyse de données issues de corpus oraux fournirait également des résultats différents de ceux issus de données à l'écrit ou obtenus par introspection. Dans une première partie, nous précisons les termes du débat concernant la dimension sémantique du passif. Nous soulignerons l'importance de prendre en considération les contraintes distributionnelles du passif par rapport à l'actif. Dans une deuxième partie, nous proposons une description des emplois de 8 verbes à l'actif et au passif dans le corpus des ESLO. Les données recueillies montrent que les deux constructions ne sont pas synonymes.

\section{Un débat dans l'analyse sémantique du passif}

\subsection{L'hypothèse classique d'une synonymie entre actif et passif}

5 Selon la conception la plus courante, « [l]es constructions passives ne modifient pas le sens dénotatif de la phrase, et leur fonction la plus générale se situe au niveau discursif» (Creissels, 2006, p.45). Autrement dit, les deux constructions sont habituellement considérées comme synonymes ${ }^{3}$.

6 Certains travaux envisagent néanmoins que actif et passif ne seraient pas synonymes, y compris sur le plan notionnel. Gaatone (1998) mentionne l'hypothèse classique selon laquelle le passif ne serait acceptable que si « le référent du sujet du passif peut être dit “affecté" par le procès désigné par le verbe » (p. 25)4. Gaatone oppose à cette hypothèse le cas de verbes comme recevoir ou subir où c'est le premier argument $\mathrm{X}$ qui est affecté par le procès et non le second argument Y. Or, selon l'auteur, ces verbes sont tout autant passivables que les verbes transitifs où c'est $Y$ qui a le rôle de patient. L'auteur donne comme exemple d'emploi passivable de recevoir la séquence suivante (p. 26) :

(2)

a. Jean a reçu un coup

b. un coup a été reçu par Jean

7 Dès lors, selon Gaatone, l'hypothèse sémantique de l'objet affecté ne tient pas et l'auteur conclut à la synonymie entre actif et passif. 


\subsection{Le problème des contraintes distributionnelles au passif}

Indépendamment de la discussion sur la synonymie ou non entre actif et passif, de nombreux auteurs ont relevé un certain nombre de cas où une phrase active, syntaxiquement transitive directe ${ }^{5}$, donne difficilement lieu à une phrase passive, comme dans les exemples suivants (cf. en particulier Ruwet, 1983; Leclère, 1993; Gaatone, 1998) :

(3)

a. Marie a arrêté l'école à 14 ans

b. Paul a regardé le match France-Écosse à la télé

c. mon voisin a quitté cette grande ville agitée sans regret

d. mon amie adore la crème au caramel

e. Nicolas habite le $11^{\mathrm{e}}$ arrondissement.

f. ce mélange sent le jasmin

g. ce cachalot pèse 15 tonnes

(4)

a. ?? l'école a été arrêtée par Marie à 14 ans

b. ? le match France-Écosse a été regardé par Paul à la télé

c. ?? cette grande ville agitée a été quittée par mon voisin sans regret

d. ?? la crème au caramel est adorée par mon amie

e. ??? Le $11^{\mathrm{e}}$ arrondissement est habité par Nicolas

f. * le jasmin est senti par ce mélange

g. * 15 tonnes sont pesées par ce cachalot

Depuis les années 1970, de nombreuses hypothèses ont été proposées pour tenter d'expliquer ces contraintes distributionnelles. Les principales sont les suivantes: une séquence serait difficilement passivable parce que i) l'emploi du verbe est plus ou moins figé ; ii) il s'agit d'un emploi figuré (Leclère, 1993) ; iii) le procès est statif (Milner, 1986) ; iv) le procès a un sens attributif (Leclère, 1993 ; Milner, 1986).

D. Gaatone (1998) montre, à partir de nombreux contre-exemples, que les trois premières hypothèses ne tiennent pas: on peut trouver de nombreux emplois figés, figurés ou statifs parfaitement passivables, respectivement illustrés par les trois exemples suivants (voir Jalenques (2015) pour 6 et 7):

(5)

a. on a mis les points sur les « $\mathrm{i}$ »

b. les points ont été mis sur les « $\mathrm{i}$ »

(6)

a. la ville de Fukushima a été touchée par un nouveau séisme [sens « concret »]

b. le peuple japonais a été touché par l'élan de solidarité international [sens figuré]

(7)

a. les deux jumeaux ont été séparés à la naissance [procès dynamique, agentif]

b. les deux pièces sont séparées par une cloison amovible [procès statif]

11 L'hypothèse de la contrainte sur les procès attributifs est plus solide (elle rend compte du blocage du passif en (4)f,g). Elle n'est cependant pas satisfaisante, car elle ne rend pas compte de tous les emplois difficilement passivables. En effet, on relève de nombreux emplois non attributifs difficilement passivables, en particulier avec des procès dynamiques, comme les exemples (4)a-c ci-avant ou avec des emplois statifs non attributifs, comme en (4)d,e.

12 Gaatone (1998) considère ainsi que «[l']étude minutieuse des données révèle un comportement hautement idiosyncrasique des verbes par rapport au passif » (p. 66). Si ces contraintes distributionnelles du passif par rapport à l'actif sont effectivement 
idiosyncrasiques, alors elles sont sans conséquence sur l'analyse du passif proprement dit.

Par ailleurs, il semble difficile d'expliquer ces contraintes sur le plan pragmaticodiscursif. En effet, comme plusieurs auteurs l'ont montré, les fonctions du passif sont très variables. Sans entrer dans les détails ici $^{6}$, notons que le deuxième argument $Y$ d'une partie au moins des emplois en (3) peut être topicalisé, par exemple avec une dislocation gauche :

(8)

a. le match France-Écosse, Paul l'a regardé à la télé

b. Cette grande ville agitée, mon voisin l'a quittée sans regret

c. Oh de la crème au caramel! Mon amie adore ça !

Dès lors, sur le plan de la structure informationnelle, rien n'empêche $Y$ de pouvoir occuper la position de sujet au passif dans ces emplois en (8). D'autre part, dans ces trois emplois, l'argument $\mathrm{X}$ peut difficilement être mis à l'arrière-plan. On pourrait alors envisager d'expliquer la contrainte d'emploi au passif en (8) à partir de l'hypothèse classique selon laquelle le passif marquerait la mise à l'arrière-plan communicatif du premier argument X. Mais, le passif n'impose pas systématiquement cette mise à l'écart de X. D'ailleurs, à l'oral, Hamma (2020) a montré que le passif pouvait au contraire marquer la focalisation de ce premier argument. En conséquence, les exemples en (8) ne contreviennent pas non plus à une contrainte générale sur le degré de saillance de $\mathrm{X}$ au passif. Si ces contraintes distributionnelles doivent recevoir une explication, ce sera au niveau sémantique (notionnel).

Il apparait ainsi que le problème de la relation sémantique entre actif et passif et le problème des contraintes distributionnelles sont liés :

- soit le passif et l'actif sont synonymes sur le plan notionnel, en particulier la relation sémantique entre le verbe et le $2^{\mathrm{e}}$ argument est identique à l'actif et au passif et dans ce cas on doit pouvoir montrer que les contraintes distributionnelles au passif sont effectivement idiosyncrasiques, irrégulières, comme le soutient Gaatone (1998);

- soit ces contraintes distributionnelles peuvent être rapportées à un fonctionnement sémantique régulier. Dans ce cas, le passif est sémantiquement plus contraint que l'actif. Dès lors, il n'y a pas synonymie entre actif et passif.

En résumé, la thèse d'une relation synonymique entre actif et passif n'est défendable que si les contraintes distributionnelles au passif sont idiosyncrasiques.

\subsection{Nécessité de combiner analyse sémantique du passif et prise en compte des contraintes distributionnelles}

Or, selon plusieurs travaux récents (Raineri, 2010; Jalenques, 2010, 2015), ces contraintes distributionnelles relèveraient effectivement d'un fonctionnement sémantique régulier. Les auteurs en déduisent que l'actif et le passif ne sont pas synonymes.

Il est indéniable qu'au moins certaines des contraintes distributionnelles au passif manifestent une différence notionnelle entre l'actif et le passif. Reprenons l'emploi (3)e associé au verbe habiter (repris à Ruwet, 1983, p. 51) :

(9)

a. Nicolas habite le $11^{\mathrm{e}}$ arrondissement.

b. ?? Le $11^{\mathrm{e}}$ arrondissement est habité par Nicolas. 

un fait général que, dans ce cas, le passif n'est naturel que si le référent du sujet (de l'actif) "occupe", en quelque sorte, "toute la place" » (p. 51). Dès lors, la séquence ci-dessus est pragmatiquement étrange. Elle sera beaucoup plus acceptable si $\mathrm{Y}$ correspond à un collectif, comme dans l'exemple suivant :

(10)

a. Un grand nombre d'étrangers habitent le $11^{\mathrm{e}}$ arrondissement.

b. Le $11^{\mathrm{e}}$ arrondissement est habité par un grand nombre d'étrangers.

Cependant, l'étrangeté sur le plan pragmatique de (9)b découle d'une différence sémantique entre actif et passif. En effet, précisément, à l'actif, on comprend que Nicolas n'habite pas tout le $11^{\mathrm{e}}$ arrondissement. Si la relation sémantique entre $\mathrm{Y}$ et habiter était la même à l'actif et au passif, et donc si les deux phrases étaient synonymes notionnellement, alors on devrait comprendre en (9)b la même chose qu'en (9)a, à savoir que Nicolas est un habitant parmi d'autres $d u 11^{\mathrm{e}}$ arrondissement ; or, ce n'est pas le cas. En conséquence, la relation sémantique entre le $2^{\mathrm{e}}$ argument $\mathrm{Y}$ et le procès n'est pas la même entre les deux phrases ; celles-ci ne sont donc pas synonymes.

21 Notons que la séquence (4)b présente une étrangeté similaire. On a l'impression que le match n'a été regardé que par Paul, ou en tout cas qu'il en a été le principal spectateur, effet sémantique que l'on n'observe pas à l'actif. Notons également que si on remplace le premier argument Paul par un collectif, là encore la séquence devient plus acceptable, comme pour habiter :

(11)

Le match France-Écosse a été regardé par plus de 15 millions de téléspectateurs.

Nous ne discuterons pas ici en détail les analyses de Raineri et de Jalenques. Précisons simplement que, au passif, selon Raineri (2010), le second argument $Y$ serait toujours affecté (au sens large) par le procès; selon Jalenques (2015), Y serait toujours « caractérisé sémantiquement par le participe passé combiné éventuellement à d'autres éléments de la proposition» (p.13). Adopter ces hypothèses revient à contester le contre-argument de Gaatone (1998) évoqué en 2.1 selon lequel des verbes comme recevoir ou subir seraient facilement passivables.

Jalenques souligne en particulier que nombre des verbes manifestant des contraintes distributionnelles au passif sont polysémiques. Or, la variation sémantique propre à ces verbes n'est généralement pas prise en considération dans les discussions sur les contraintes distributionnelles. Pour un certain nombre de verbes, cette variation sémantique met en jeu une variation de portée sémantique du procès, illustrée par les deux emplois suivants du verbe arrêter :

(12)

a. la médecine du travail a arrêté mon frère pour 15 jours

b. ce médecin a arrêté la cigarette en 2012

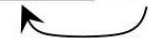

En (12)a, le changement de situation exprimé par arrêter porte sur le second argument (il ne peut plus travailler); le procès porte sémantiquement sur Y. Au contraire, en (12)b, le procès porte sémantiquement sur le premier argument. Cette fois-ci, c'est la situation du médecin (X) qui change (il n'est plus fumeur). L'auteur constate que l'emploi pour lequel le procès porte sémantiquement sur $Y$ est nettement plus acceptable que celui où le procès porte sur $\mathrm{X}$ : 
(13)

a. mon frère a été arrêté par la médecine du travail pour 15 jours

b. ?? en 2012, la cigarette a été arrêtée par ce médecin distributionnelles au passif se fonde sur des données construites par introspection ou bien sur des données tirées de corpus écrits. Nous proposons ici de tester la validité des différentes observations évoquées ci-avant, en utilisant le cadre d'analyse de Jalenques (2015). On le sait, il arrive souvent que des séquences jugées par introspection comme peu ou pas acceptables puissent s'observer à l'oral. Inversement, il arrive aussi que des séquences jugées intuitivement acceptables ne se rencontrent pas dans les données attestées à l'oral. Il s'agira ainsi de vérifier si les séquences attestées au passif à l'oral privilégient effectivement les cas où le procès porte sémantiquement sur le second argument $Y$ ou pas.

\section{Observations sémantiques sur 8 verbes au passif dans le corpus des ESLO}

\subsection{Choix du corpus et des verbes examinés}

Nos observations ont été faites à partir de données extraites du corpus des ESLO qui a été retenu pour deux raisons: d'une part pour sa taille, il constitue, à notre connaissance, le plus grand corpus de données de français oral (de France) à ce jour. La fréquence du passif à l'oral étant relativement faible, il était important de disposer d'un corpus suffisamment grand pour espérer récupérer des données quantitativement significatives. D'autre part, nous avons choisi ce corpus en raison de la diversité des situations de parole représentées (non seulement des entretiens avec des questionnaires standardisés, mais aussi des situations de parole plus spontanée, lors de repas ou des paroles captées dans la rue, les transports publics, les commerces, etc.), ce qui limite les biais éventuels liés à un type d'interactions trop spécifique.

Nos observations concernent 8 verbes: recevoir, subir, supporter, arrêter, habiter, comporter, composer, constituer. Pour chaque verbe, nous avons extrait l'ensemble de ses emplois à toutes les formes conjuguées attestées dans l'ensemble du corpus. Pour les 
5 premiers verbes, l'extraction a été faite par une requête sur le logiciel TXM, à partir de la version étendue du corpus des ESLO, disponible au Laboratoire ligérien de linguistique (version du corpus constituée de 4887521 segments) $^{7}$. Pour les 3 derniers verbes (comporter, composer, constituer), l'extraction a été faite directement sur le portail internet du corpus en ligne (<http://ESLO.huma-num.fr/index.php>), sur l'ensemble des enregistrements accessibles sur le site (2 560794 segments) ${ }^{8}$.

Le choix de ces 8 verbes a résulté de la combinaison de plusieurs critères. Tout d'abord, nous avons retenu des verbes qui étaient déjà discutés dans la littérature sur les contraintes distributionnelles, puisque l'objectif principal de cet article est de tester la validité de ces données construites vis-à-vis de données attestées orales. D'autre part, nous avons retenu des verbes dont le nombre d'occurrences dans les ESLO est suffisamment important pour que les données quantitatives soient significatives, et suffisamment modeste pour permettre le classement sémantique «à la main » de tous ces emplois.

31 Nous avons choisi les verbes recevoir, subir et supporter car l'étude de leurs emplois au passif est cruciale dans le débat avec Gaatone (1998) sur la synonymie entre actif et passif. Nous avons parailleurs retenu le verbe arrêter car sa polysémie est particulièrement nette du point de vue de la variation de portée sémantique du procès. Dans le cadre des verbes ayant des emplois statifs, nous avons retenu le verbe habiter souvent discuté dans la littérature sur les contraintes distributionnelles. Enfin, nous avons retenu les verbes comporter, composer, constituer évoqués par Gaatone (1998) car, bien que sémantiquement proches, ils ont un comportement différent au passif.

Ces 8 verbes représentent un total de 4316 occurrences examinées manuellement. Nous y avons relevé 194 emplois au passif périphrastique, ce qui représente $4,5 \%$ du total des emplois. Ce dernier chiffre doit être pris avec précaution dans la mesure où certains verbes ont proportionnellement peu d'emplois transitifs directs, donc peu d'emplois susceptibles de donner lieu à un passif. Par exemple, le verbe arrêter a 51 occurrences au passif sur un total de 1078 occurrences. Cependant, sur ces 1078 occurrences, le verbe n'a que 161 occurrences correspondant à un emploi transitif direct. Les phrases passives représentent donc en réalité $24 \%$ des emplois transitifs directs de ce verbe.

Nous n'avons pas la place de discuter ici la question de la transitivité sémantique. Observons simplement que dans les ESLO la proportion d'emplois au passif d'un verbe n'est pas proportionnelle au degré de transitivité sémantique de ce verbe. Par exemple, un verbe comme regarder, transitif direct dans la plupart de ses emplois, correspondant à un procès dynamique et agentif, a un fonctionnement sémantique proche du prototype de transitivité sémantique. Pourtant, sur 2575 occurrences dans l'ensemble du corpus des ESLO, ce verbe n'a en tout et pour tout que 3 occurrences au passif. Inversement, un verbe comme composer, dont plus de la moitié des emplois transitifs directs correspondent à un procès non agentif et statif, et qui n'a que 52 occurrences dans l'ensemble du corpus, présente néanmoins 20 occurrences au passif.

\subsection{Le verbe recevoir et quelques synonymes}

Commençons par le verbe recevoir dont l'acceptabilité au passif remet en cause, selon Gaatone (1998), l'hypothèse selon laquelle le passif serait sémantiquement plus contraint que l'actif (cf. discussion en 2.1). Nous avons étendu les observations à deux 
synonymes du verbe recevoir, à savoir les verbes subir et supporter dont le premier argument $\mathrm{X}$ a également un rôle sémantique de patient, de façon privilégiée.

\subsubsection{Le verbe recevoir}

Nous avons relevé dans notre corpus 832 occurrences du verbe recevoir. D'après les séquences relevées, on peut répartir les emplois de recevoir à l'actif en 5 acceptions. Pour chacune, nous donnons un exemple tiré du corpus :

- a1 : « accueillir des personnes » :

(14)

quand je reçois de la famille et qu'il y a des enfants on va au parc floral

- a2 : « être destinataire d'un message, d'un document, d'un objet, d'un appel téléphonique » :

(15)

euh eh ben on reçoit l'Équipe nous recevons le la République du Centre bien sûr oui

- a3 : « être bénéficiaire d'un enseignement / une formation / une éducation » :

(16)

euh moi je reconnais que j'ai reçu aucune formation euh spéciale pour enseigner

- a4 : « qqch nous apporte émotionnellement » (dans le cotexte, recevoir est opposé à donner) :

(17)

je pense que il y a deux mouvements il y a ce que l'on reçoit des autres et ce que l'on peut

donner soi-même à sa ville

- a5 : « être l'objet d'une action, d'un évènement (associée au second argument) » :

(18)

il avait reçu des plombs dans l'œeil cet hiver à la chasse ça s'est passé en décembre ou janvier cette affaire

De l'acception a1 à l'acception a5, on observe une variation importante du rôle sémantique du premier argument $\mathrm{X}$. Pour a1 (accueillir des personnes), le premier argument a clairement un rôle agentif. Au contraire, pour a5 (" être l'objet d'une action, d'un évènement $»)$, le premier argument a un rôle de patient. On relève une sixième acception, observée uniquement au passif :

- a6 : « valider la réussite de quelqu'un à une formation » :

(19)

donc j'ai passé le concours pour euh pour être élève à l'école du Louvre et j-et j'ai donc j'ai étét-reçue

Sur les 832 occurrences de recevoir, on relève ainsi 704 occurrences à l'actif et 90 occurrences au passif ${ }^{9}$. Le verbe recevoir se rencontre donc au passif, comme le signale Gaatone. Cependant, examinons le nombre d'occurrences du verbe pour chaque acception, à l'actif et au passif :

Tableau 1. - Nombre d'occurrences du verbe recevoir pour chaque acception, à l'actif et au passif.

\begin{tabular}{|c|c|c|c|c|c|c|c|}
\hline & $\begin{array}{c}\text { a1 } \\
\text { accueillir des } \\
\text { personnes }\end{array}$ & $\begin{array}{c}\text { a2 } \\
\text { être destinataire } \\
\text { d'un message, } \\
\text { d'un objet, d'un } \\
\text { appel } \\
\text { téléphonique }\end{array}$ & \begin{tabular}{|c|} 
a3 \\
être \\
bénéficiaire \\
d'un \\
enseignement / \\
une formation / \\
une éducation
\end{tabular} & $\begin{array}{c}\text { a4 } \\
\text { « qqch nous } \\
\text { apporte } \\
\text { émotionnelle- } \\
\text { ment » }\end{array}$ & $\begin{array}{c}\text { a5 } \\
\text { être l'objet } \\
\text { d'une action, } \\
\text { d'un évènement }\end{array}$ & \begin{tabular}{|c|}
$\mathrm{a} 6$ \\
" valider la \\
réussite de \\
quelqu'un à une \\
formation »
\end{tabular} & TOTAL \\
\hline ACTIF & 236 & 382 & 45 & 10 & 31 & 0 & 704 \\
\hline PASSIF & 47 & 3 & 0 & 0 & 0 & 40 & 90 \\
\hline
\end{tabular}


Cela donne le graphique suivant :

Figure 1. - Nombre d'occurrences pour chaque acception du verbe recevoir.

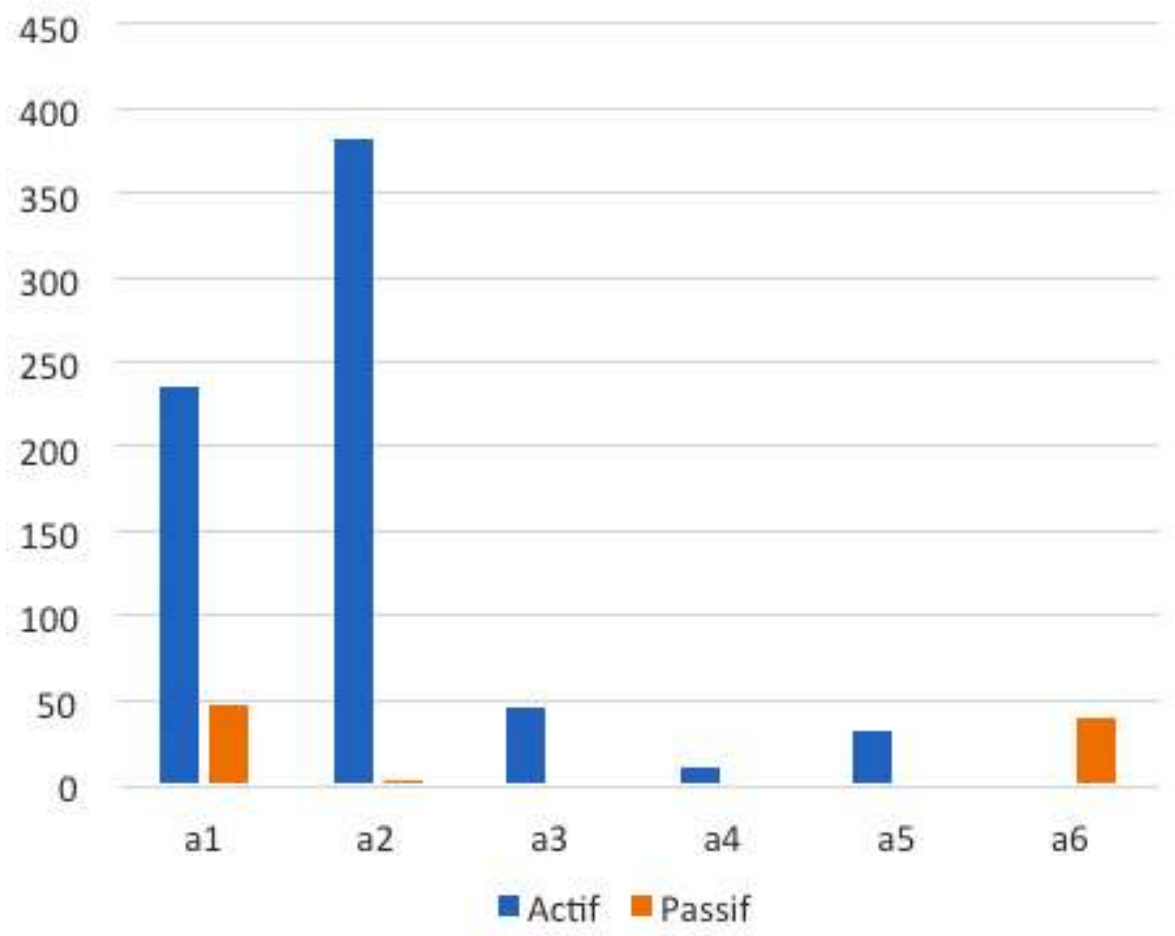

La répartition des acceptions du verbe recevoir apparait bien différente à l'actif et au passif. En particulier, l'acception «être destinataire d'un message, d'un objet, d'un appel téléphonique » est quasi absente des emplois au passif alors qu'elle correspond à l'emploi le plus fréquent à l'actif. Deux points en particulier sont à observer : en diathèse active, l'acception privilégiée n'est pas celle où le premier argument est le plus agentif (acception a1). Au contraire, au passif, cette acception a1 est privilégiée. Les acceptions $\mathrm{a} 3$ à $\mathrm{a} 5$ où le procès porte sémantiquement sur le premier argument $\mathrm{X}$ sont totalement absentes au passif. Il apparait donc que le passif filtre la polysémie du verbe recevoir et que l'acception a1 est nettement privilégiée par rapport à l'acception a5 supposée tout aussi passivable selon Gaatone (1998).

En outre, ces résultats sont concordants avec des observations de Jalenques (2010) sur corpus écrit. L'auteur a notamment examiné les occurrences du verbe recevoir dans le corpus Frantext ${ }^{10}$. L'auteur a examiné 200 occurrences du verbe en diathèse active. Il apparait que les emplois correspondant à l'acception a1 représente $13 \%$ des emplois tandis que l'acception a6 est absente. Au contraire, au passif, sur 263 occurrences, l'auteur relève $62 \%$ d'emplois correspondant à l'acceptiona1 et $28 \%$ pour l'acception a6. Il apparait donc dans ce corpus écrit comme dans le corpus des ESLO que les deux acceptions quantitativement dominantes sont les mêmes, à savoir a1 et a6, c'est-à-dire les acceptions où le procès porte le plus sémantiquement sur Y.

\subsubsection{Les verbes subir et supporter}

41 Venons-en aux verbes subir et supporter, sémantiquement proches du verbe recevoir $\mathrm{du}$ point de vue du rôle sémantique de $\mathrm{X}$. On relève 91 occurrences du verbe subir. La 
polysémie de ce verbe est plus restreinte que celle de recevoir. En particulier, on peut paraphraser l'ensemble de ses emplois par une formulation du type «être l'objet sur lequel s'exerce une action, un évènement " (subir une opération à cœur ouvert / subir un interrogatoire, etc.) ; c'est-à-dire un sens proche de celui correspondant à l'acception 5 de recevoir. Nous venons de voir que recevoir n'a aucun emploi attesté au passif pour cette acception. Il apparait que le verbe subir n'a lui non plus aucun emploi attesté au passif dans les ESLO.

Le verbe supporter offre 108 occurrences. Dans Le Grand Robert de la langue française (version informatisée), les principales acceptions de ce verbe sont: 1) « recevoir le poids, la poussée de (qqch.) sur soi, en maintenant»; 2) «subir, éprouver les conséquences pénibles de (un évènement, un état, une action) sans faiblir, avec constance.» Nous constatons que ces définitions mettent en jeu respectivement l'acception 5 du verbe recevoir et le sens du verbe subir. On observe que le verbe supporter n'a qu'un seul emploi attesté au passif :

(20)

pour ce que les dépenses importantes euh d'une activité soient aussi supportées par d'autres activités et qu'elle soient acceptées (ESLO1_INTPERS_423)

43 L'interprétation de cette séquence n'est pas très claire, mais, d'après l'enregistrement sonore, il s'agit d'un emploi sémantiquement proche de soutenir, c'est-à-dire un emploi proche de l'anglicisme supporter (to support: "soutenir »), autrement dit un emploi où l'interprétation du premier argument du verbe est plus proche du rôle agentif et le rôle $\mathrm{du}$ second argument plus proche d'un patient. Le procès porte donc sémantiquement sur le second argument $Y$. Àl'actif, nous avons relevé 3 occurrences de ce type (supporter une équipe de foot).

44 Nous avons examiné également les emplois du verbe endurer, mais le très faible nombre d'occurrences ne permet pas de dégager de tendance (seulement 2 occurrences, à l'actif). Concernant les trois verbes recevoir, subir et supporter, il apparait clairement que l'emploi du passif est très limité ou absent lorsque c'est le premier argument qui est interprété comme un patient.

Ces données attestées contredisent donc les données construites proposées par Gaatone (1998) à l'appui de l'idée selon laquelle les emplois au passif avec un argument $\mathrm{X}$ ayant le rôle de patient se rencontre aussi facilement au passif que les cas où $\mathrm{X}$ a un rôle d'agent. Il apparait clairement que le passif contraint le rôle sémantique du premier argument.

\subsection{Le verbe arrêter}

Le verbe arrêter a 1078 occurrences dans le corpus des ESLO. La plupart de ses emplois correspondent à des emplois transitifs indirects (il ne faut pas s'arrêter aux apparences) ou bien à des emplois réflexifs (je m'arrête là). Ceci dit, le verbe présente également 161 occurrences correspondant à des emplois transitifs directs qui peuvent être réparties en quatre acceptions.

- a1 : « mettre un terme à la liberté de qqn » :

(21)

il a bien entendu fait arrêter le général Perré il l'a fait mettre en prison pendant six mois (ESLO1_INTPERS_440)

- a2 : «stopper ou interrompre quelqu'un dans un processus, une activité » : 
(22)

c'est pas lui hein c'est euh la la la médecine du travail qui l'arrête (ESLO2_ENT_1041)

- a3 : «stopper un processus, un objet dynamique» :

(23)

a. alors le médecin a dit [...] j'ai arrêté la gangrène (ESLO1_REPAS_270)

b. eh bien je vais arrêter la machine alors (ESLO1_ENT_057)

- a4 : « mettre un terme à son engagement dans une activité, une situation » :

(24)

a. il avait il avait arrêté l'école à quatorze ans (ESLO1_ENT_013)

b. pour des raisons euh familiales j'ai arrêté le Rotary pendant dix ans (ESLO2_ENT_1064)

Dans les trois premières, le procès porte sémantiquement sur Y (le second argument). Au contraire, dans la dernière, le procès porte sémantiquement sur le premier argument. Quand un élève arrête l'école, cela renvoie à un changement de situation pour l'élève $(\mathrm{X})$ et non pour l'école $(\mathrm{Y})$. On relève également deux hapax, correspondant respectivement à l'acception a5 : "fixer une limite à un objet » (à l'actif) et a6 " choisir, décider » (au passif), correspondant aux deux exemples suivants :

(25)

non parce que là-bas ils ont arrêté la cloison différemment mais y a des fenêtres qui arrivaient au ras des cloisons et c'est des fenêtres oscillo-battantes (ESLO2_ENT_1049)

(26)

y a un timing qui est prévu et si euh le parfum n'est pas n'est pas arrêté ça sortira pas en développement (ESLO2_ENT_1054)

Les emplois au passif sont au nombre de 50 (+ l'hapax en (26)). Observons le nombre d'occurrences pour chaque acception, à l'actif et au passif :

Tableau 2. - Nombre d'occurrences pour chaque acception du verbe arrêter, à l'actif et au passif.

\begin{tabular}{|l|l|l|l|l|l|}
\hline & a1 & a2 & a3 & a4 & TOTAL \\
\hline ACTIF & 7 & 13 & 84 & 56 & 160 \\
\hline PASSIF & 9 & 33 & 8 & 0 & 50 \\
\hline
\end{tabular}

Figure 2. - Nombre d'occurrences pour chaque acception du verbe arrêter.

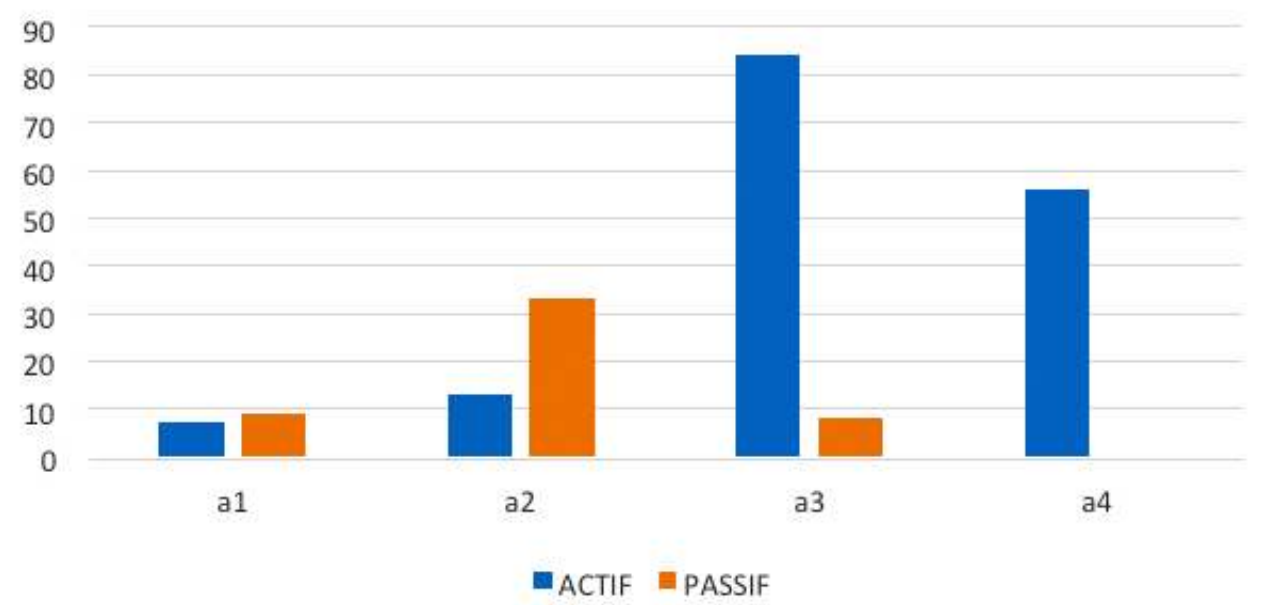


Au contraire, avec l'acception a1 «mettre un terme à la liberté de qqn », pourtant peu fréquente en diathèse active ( 7 occurrences), on relève 9 occurrences au passif, c'est-àdire davantage qu'à l'actif. Idem pour l'acception a2. Le poids respectif de chaque acception est nettement différent entre la diathèse active et la diathèse passive ; de plus, la diversité des acceptions observée au passif est plus restreinte que la diversité des acceptions à l'actif (absence de l'acception a4). Là encore, le passif semble filtrer la polysémie du verbe observée à l'actif en privilégiant les emplois où le procès porte sémantiquement sur Y.

\subsection{Observations de quelques emplois statifs}

\subsubsection{Le verbe habiter}

Abordons le verbe habiter dont la contrainte d'emploi au passif a été discutée par plusieurs auteurs (cf. Ruwet, 1983, p. 51; Gaatone, 1998, p. 260 ; Raineri, 2010, p. 60 ; Jalenques, 2015, p. 10-11). Selon ce dernier auteur, ce verbe correspond à une portée sémantique du procès sur $X$, dans ses emplois courants. Dans l'exemple 9 discuté en 2.3, le procès décrit la situation de Nicolas (X) et non la situation du $11^{\mathrm{e}}$ arrondissement (Y). À priori, ce verbe est donc difficilement passivable.

On relève 1968 occurrences du verbe habiter dans le corpus des ESLO. Les emplois transitifs directs sont minoritaires; ils représentent $26 \%$ des emplois (520 occurrences). De façon générale, la variation sémantique du verbe habiter peut être ramenée à deux grandes classes d'emplois, la seconde classe donnant lieu à des emplois facilement passivables :

a. sens « concret » : avoir sa demeure / vivre habituellement quelque part;

b. sens figuré : être fortement présent dans l'esprit ou dans le cœur de quelqu'un (le démon du jeu l'habite / il est habité par le démon du jeu). Notons qu'ici le procès caractérise la situation dans laquelle se trouve la personne considérée ; il porte donc sémantiquement sur $Y$.

Dans le corpus des ESLO, tous les emplois transitifs directs correspondent à l'acception a). En voici un exemple :

(27)

Marion ha- habite le quartier avec mon fils hein et euh juste au coin de la rue oui j'ai toujours habité le quartier (ESLO2_ENT_1004)

Face à ces 520 occurrences transitives directes, on relève seulement 9 occurrences au passif. Nous avions vu en 2.3 que, selon Ruwet (1983), le passif avec cet emploi est difficilement acceptable, sauf si X correspond à un collectif. De fait, les 9 emplois

Lidil, 64 | 2021 
attestés au passif correspondent tous à un premier argument $\mathrm{X}$ collectif ou générique, comme dans les 3 exemples suivants :

(28)

alors vous avez tout ce ce noyau de de ville là euh n'était habité que par des gens comme ça hm hm maintenant euh ça a changé (ESLO1_ENT_056)

(29)

toute proportion gardée euh si si ce quartier est habité par des Orléanais de souche même

d'origine plus modeste euh je pense que les réactions sont à peu près les mêmes (ESLO1_ENT_119)

(30)

on a été le voir les logements qui se construisent par là mais c'est pas encore tout tout

habité hein ça se construit (ESLO1_REPAS_272)

C'est cette dimension collective de X qui permet une caractérisation de Y (on apporte une précision à propos du noyau de la ville, du quartier). Par contraste, les emplois actifs mettent presque toujours en jeu un premier argument $\mathrm{X}$ non collectif (495 occurrences sur 520), comme dans l'exemple (9a). Les données intuitives présentées par Ruwet (1983) sont donc confirmées par ces données attestées orales.

\subsubsection{Les verbes comporter, composer, constituer}

57 Abordons maintenant les verbes comporter, composer et constituer. À partir de données issues de l'introspection, Gaatone (1998, p. 106) observe que le verbe comporter est difficilement passivable, à la différence des verbes comme composer ou constituer. L'auteur propose les deux exemples suivants :

(31) les légumes et les laitages composent l'essentiel du menu (Gaatone, 1998, p. 106)

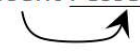

(32)

cet ouvrage comporte plusieurs parties

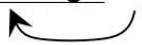

D'après Jalenques (2015), on peut concevoir la différence entre ces verbes à nouveau comme une différence de portée sémantique du procès. En effet, en (31), le procès décrit le menu (il précise de quoi est fait le menu), il ne décrit pas comment sont les légumes ni les laitages. Le procès porte donc sémantiquement sur Y. Au contraire, en (32), le procès décrit comment est l'ouvrage, c'est-à-dire le premier argument $\mathrm{X}$ (il décrit de quoi est fait l'ouvrage). On constate à nouveau que lorsque le procès porte sur $\mathrm{Y}$, le passif est acceptable, alors que lorsque le procès porte sur $\mathrm{X}$, il est difficilement acceptable :

(33)

a. l'essentiel du menu est composé de légumes et de laitages

b. ?? plusieurs parties sont comportées par cet ouvrage

Dans les ESLO, nous avons relevé 71 occurrences du verbe comporter, dont 36 transitives directes. Nous n'avons relevé aucune occurrence au passif pour ce verbe, confirmant l'intuition de Gaatone. De son côté, le verbe composer a 52 occurrences dans le corpus, dont 13 occurrences en construction transitive directe. Sur ces 13 emplois, 6 correspondent à un procès dynamique et 7 correspondent à un procès statif. Malgré le nombre peu important d'emplois transitifs directs par rapport à comporter, le verbe composer a 20 emplois au passif, c'est-à-dire davantage que ses emplois transitifs directs à l'actif ${ }^{11}$. Sur ces 20 occurrences, on relève deux emplois correspondant à un procès 
dynamique, toutes les autres occurrences correspondant à un procès statif, comme dans les exemples ci-dessous :

(34)

a. notre clientèle est presqu'exclusivement composée de très grandes firmes (ESLO1_INTPERS_420)

b. bah il faut dire que l'équipe était composée de gens absolument disparates

(ESLO1_INTPERS_448)

Notons que dans ces deux exemples la présence du complément dit d'agent est obligatoire.

Comme pour le verbe composer, les emplois du verbe constituer correspondent généralement à une portée sémantique du procès sur $Y$. On relève 110 occurrences dont 23 au passif pour 67 emplois en construction transitive directe. Les données attestées dans les ESLO montrent donc une forte proportion de passifs pour les verbes composer et constituer et aucun passif pour le verbe comporter.

\section{Bilan}

61 Les résultats de ces premières observations tirées du corpus des ESLO sont contrastés. D'un côté, ils contredisent les données de Gaatone (1998), issues de l'introspection, sur les verbes recevoir et subir. En particulier, les emplois attestés au passif de recevoir privilégient clairement les acceptions pour lesquels le procès porte sémantiquement sur le second argument du verbe. On observe en outre une convergence remarquable entre les données sur ce verbe issues de corpus oral et de corpus écrit. D'un autre côté, les données issues de l'introspection concernant les verbes arrêter, habiter, comporter, composer, constituer sont corroborées par les données attestées orales.

Les contraintes distributionnelles au passif apparaissent sémantiquement régulières. Ainsi, l'étude des 8 verbes examinés dans le corpus des ESLO montre que le passif privilégie systématiquement les emplois où le procès porte sémantiquement sur le second argument du verbe, quel que soit le verbe. Il est crucial de noter que cette situation n'est pas symétrique de l'actif. En effet, en diathèse active, le procès peut aussi bien porter sémantiquement sur l'argument $\mathrm{X}$ que sur l'argument $\mathrm{Y}$. Cela dépend essentiellement des propriétés lexicales du verbe. Ainsi, à l'actif, avec recevoir, le procès porte sémantiquement sur $\mathrm{X}$ dans la majorité des occurrences observées alors qu'avec arrêter, en construction transitive directe, le procès porte sur $\mathrm{Y}$ dans la majorité des occurrences observées.

Lorsqu'un verbe est polysémique et admet, à l'actif, à la fois des emplois avec portée sémantique du procès sur $\mathrm{X}$ et des emplois avec portée sur $\mathrm{Y}$, le passif filtre cette polysémie et sélectionne les emplois avec portée sémantique du procès sur $\mathrm{Y}$ ( $\mathrm{cf}$. arrêter). Les emplois du passif apparaissent donc sémantiquement plus contraints que ceux de l'actif. Les deux constructions ne sont donc pas synonymes, y compris sur le plan notionnel, contrairement à l'hypothèse la plus courante.

64 Les différences entre actif et passif ne se réduisent donc pas à des différences d'ordre pragmatico-discursif. La spécificité du passif périphrastique en français se manifeste sur deux plans : pragmatico-discursif et sémantique. Les contraintes distributionnelles dégagées ici relèvent spécifiquement du niveau sémantique. Bien sûr, ces premiers résultats devront être consolidés par l'analyse sémantique d'un plus grand nombre de verbes à l'oral. 


\section{BIBLIOGRAPHIE}

BlanCHe-BenVEniste, Claire. (2000). Analyse de deux types de passifs dans les productions de français parlé. Dans L. Schoesler (dir.), Le passif : actes de colloque (Études romanes, 45, p. 303-319). Museum Tusculanum Press.

Bolinger, Dwight. (1975). On the passive in English. Dans A. Makkai \& V. B. Makkai (dir.), The First Lacus Forum (p. 57-80). Hornbeam Press.

CARLIER, Anne. (2002). Les propriétés aspectuelles du passif. Cahiers Chronos, 10, 41-63.

CREISSELS, Denis. (2006). Passif et autres mécanismes de destitution du sujet. Dans D. Creissels, Syntaxe générale. Une introduction typologique (vol. 2, p. 43-57). Lavoisier.

DRUETTA, Ruggero. (2020). Le passif à l'oral. Phénoménologie et propriétés aspectuelles dans « OFROM ». Studia Linguistica Romanica, 4, 150-174.

GAATONE, David. (1998). Le passif en français. Duculot.

HAMmA, Badreddine. (2020). Variations diamésiques autour du passif périphrastique en français. Dans S.-S. Rodríguez Somolinos \& G.-J. Ferary (dir.), Marques d'oralité et représentation de l'oral en français (p. 43-66). Presses universitaires de Savoie.

HAMmA, Badreddine, TARDIF, Amélie \& BADIN, Flora. (2017). Le passif à l'oral. Fiche du Projet FRACOV (Français contemporain vernaculaire). <www.ortolang.fr/market/corpora/fracov? path $=\% 2 \mathrm{~F}>$.

HELLAND, Hans Petter. (2002). Le passif périphrastique en français moderne. Museum Tusculanum Press.

JALENQUES, Pierre. (2010). Un exemple d'interaction morphologie-syntaxe-sémantique : les contraintes sur les procès transitionnels au passif périphrastique. Dans C. Alvarez Castro et coll. (dir.), Études sur la combinatoire et la hiérarchie des composants : actes du VIII ${ }^{e}$ Congrès international de linguistique française (p. 409-425). Peter Lang.

JALENQUES, Pierre. (2015). Le passif en français et les contraintes distributionnelles des verbes. Corela, 13(1). <https://doi.org/10.4000/corela.4015>.

JALENQUES, Pierre. (2016). Le passif en français et le statut référentiel du sujet. Congrès mondial de linguistique française - CMLF 2016. <https://doi.org/10.1051/shsconf/20162714001>.

JALENQUES, Pierre. (2017). Pour une analyse sémantique du passif périphrastique en français. ÉLA, 187, 325-338.

LECLÈRE, Christian. (1993). Classes de constructions directes sans passif. Langages, 109, 7-31.

MILNER, Jean-Claude. (1986). Introduction à un traitement du passif. Université Paris 7.

MULLER, Claude. (2017). Imperfections et lacunes des passifs du français. ÉLA, 187, 283-296.

RAINERI, Sophie. (2010). Analyse contrastive français-anglais du passif dans une perspective constructionnelle : sens et fonction de BE Ven, ÊTRE Vé, GET Ven, SE FAIRE Ver (Thèse de doctorat). Université Sorbonne Nouvelle - Paris 3.

RUWET, Nicolas. (1983). Du bon usage des expressions idiomatiques dans l'argumentation en syntaxe générative. Recherches linguistiques, 11, 5-84. 


\section{NOTES}

1. Le corpus des ESLO (Enquêtes sociolinguistiques à Orléans) est "composé d'enregistrements sonores et de leurs transcriptions réalisées à Orléans entre 1968 et 1974 (ESLO1) et à partir de 2008 (ESLO2)» (ESLO.huma-num.fr).

2. L'expression passif périphrastique est utilisée notamment par Gaatone (1998) et Helland (2002).

3. Les différences sémantiques liées à la présence de quantificateurs (du type tous les étudiants ont lu au moins un livre / au moins un livre a été lu par tous les étudiants), relèvent de contraintes générales sur la portée des quantificateurs, indépendantes de la spécificité du passif (Gaatone, 1998). De même, les différences sémantiques liées aux relations anaphoriques entre les arguments du verbe (du type François a perdu un œil / un œil a été perdu par François, où le lien anaphorique entre François et œil est bloqué au passif), relèvent de contraintes générales liées aux positions syntaxiques des arguments du verbe et non d'une différence sémantique entre actif et passif (cf. Jalenques, 2016). Par ailleurs, nous ne revenons pas sur la différence sémantique dans le domaine grammatical, aspectuel, entre actif et passif. Cette différence a été largement discutée dans la littérature (cf. parmi beaucoup d'autres Carlier (2002) et, pour une analyse récente sur des données orales, Druetta (2020)).

4. À notre connaissance, cette hypothèse sémantique a été initialement proposée par Bolinger (1975), pour le passif en anglais.

5. On pourrait certes discuter du degré de transitivité syntaxique de certaines de ces phrases. Il s'avère que le problème n'est pas là, dès lors que le verbe admet un complément argumental direct et nominal (pour une discussion plus détaillée, voir Jalenques, 2015, p. 5-6). En effet, des verbes clairement transitifs (syntaxiquement) comme quitter ou subir sont difficilement passivables tandis que des verbes faiblement transitifs comme vivre ou pleurer sont passivables (le confinement a été mal vécu par la population / le chanteur a été pleuré par la France entière).

6. Pour une discussion plus détaillée, voir Jalenques (2017).

7. Je remercie chaleureusement B. Hamma pour son aide précieuse dans l'extraction de ces données à partir de l'outil de fouille textuelle TXM.

8. Communication personnelle de B. Hamma.

9. La différence entre les chiffres de 832 et $794(704+90)$ découle de quelques emplois de recevoir comme participe passé employé nu (exemple : des plaintes $d$-reçues par les particuliers) et d'énoncés où le verbe est employé sans complément direct.

10. Sous-corpus des textes postérieurs à 1970, correspondant à 330 ouvrages.

11. Blanche-Benveniste (2000) avait déjà noté que «contrairement aux idées reçues, certains verbes sont plus fréquemment attestés dans leur emploi passif que dans l'actif correspondant » (p. 304).

\section{RÉSUMÉS}

Nous reprenons l'analyse sémantique du passif en français, appelé parfois passif périphrastique, à partir de données généralement marginalisées, à savoir les restrictions distributionnelles par rapport à l'actif. Par exemple, le verbe polysémique arrêter est passivable dans certains emplois (La police a arrêté un suspect / un suspect a été arrêté par la police), mais pas dans d'autres (Marie a arrêté l'école à 14 ans / ?? l'école a été arrêtée par Marie à 14 ans). Ces observations reposent généralement sur des données issues de l'introspection ou bien de corpus écrits. Nous reprenons 
l'analyse de ces contraintes distributionnelles à partir de données attestées orales, tirées du corpus des ESLO. Les premiers résultats, portant sur 8 verbes, montrent que le passif privilégie systématiquement les emplois où le procès caractérise sémantiquement le second argument du verbe. Ainsi, le passif ne serait pas synonyme de la construction active, contrairement à l'hypothèse la plus couramment admise.

We resume the semantic analysis of the passive construction in French, sometimes called passif périphrastique, based on generally marginalized data, namely restricted distributions in relation to the active construction. For example, the polysemous verb arrêter is passivable in some uses (La police a arrêté un suspect / un suspect a été arrêté par la police), but not in others (Marie a arrêté l'école à 14 ans / ?? l'école a été arrêté par Marie à 14 ans). These observations are generally based on data from invented examples or from written corpora. We have taken up the analysis of these restricted distributions using oral attested data, which was drawn from the ESLO corpus. The initial results, covering 8 verbs, show that the passive systematically favors uses in which the process semantically characterizes the second argument of the verb. Thus, the passive would not be synonymous with the active construction, contrary to the most commonly accepted assumption.

\section{INDEX}

Mots-clés : passif, sémantique, contraintes distributionnelles, polysémie des verbes

Keywords : passive construction, semantics, distributional gaps, verbal polysemy

\section{AUTEUR}

PIERRE JALENQUES

Université de Rouen, Laboratoire Dynamique du Langage In Situ 